\title{
Levels, Trends, and Inequalities in Using Institutional Delivery Services in Low- and Middle-Income Countries: A Stratified Analysis by Facility Type
}

\author{
Md. Mehedi Hasan, ${ }^{a, b}$ Ricardo J. Soares Magalhaes, ${ }^{c, d}$ Yaqoot Fatima, ${ }^{a, e}$ Saifuddin Ahmed, ${ }^{\text {f,g }}$ \\ Abdullah A. Mamun ${ }^{a, b}$
}

\section{Key Findings}

- Progress toward improving the utilization of institutional delivery services was not uniform across low- and middle-income countries (LMICs) and across subpopulations within LMICs irrespective of public and private health facilities.

- Wealth, place of residence, and education-based inequalities in the utilization of institutional delivery services are widening in many LMICs, which warrants the attention of policy makers for further investments and policy reviews.

\section{Key Implications}

- Program managers and policy makers should give special priority to people who are poorest, live in rural areas, and have low education when designing appropriate interventions for increasing institutional delivery service coverage, irrespective of public and private facilities.

- Appropriate and tailored interventions covering the disadvantaged countries and marginalized populations within countries may help countries to achieve the global target of "leaving no one behind" for the utilization of institutional delivery services by 2030.

\footnotetext{
a Institute for Social Science Research, The University of Queensland, Indooroopilly, Queensland, Australia.

b ARC Centre of Excellence for Children and Families over the Life Course (The Life Course Centre), The University of Queensland, Indooroopilly, Queensland, Australia.

' UQ Spatial Epidemiology Laboratory, School of Veterinary Science, The University of Queensland, Gatton, Australia.

d UQ Children's Health and Environment Program, Child Health Research Centre, The University of Queensland, South Brisbane, Australia.

e Centre for Rural and Remote Health, James Cook University, Mount Isa, Australia.

f Department of Population, Family and Reproductive Health, Johns Hopkins Bloomberg School of Public Health, Baltimore, MD, USA.

9 Bill and Melinda Gates Institute for Population and Reproductive Health, Johns Hopkins Bloomberg School of Public Health, Baltimore, MD, USA.

Correspondence to Md. Mehedi Hasan (m.m.hasan@uqconnect.edu.au).
}

\section{ABSTRACT}

Introduction: To ensure equitable and accessible services and improved utilization of institutional delivery it is important to identify what progress has been achieved, whether there are vulnerable and disadvantaged groups that need specific attention and what are the key factors affecting the utilization of institutional delivery services. In this study, we examined levels, trends, and inequalities in the utilization of institutional delivery services in low- and middle-income countries.

Methods: We used nationally representative cross-sectional data from Demographic and Health Surveys (DHS) conducted during 1990-2018. Bayesian linear regression analysis was performed. Results: Among 74 countries, the utilization of institutional delivery services ranged from $23.7 \%$ in Chad to $100 \%$ in Ukraine and Armenia (with $>90 \%$ in 19 countries and $<50 \%$ in 13 countries) during the latest DHS rounds. Trend analysis in 63 countries with at least 2 surveys showed that the utilization of institutional delivery services increased in 60 countries during 1990-2018, with the highest increase being in Cambodia (18.3\%). During this period, the utilization of institutional delivery services increased in $90.3 \%$ of countries among the richest, $95.2 \%$ of countries in urban, and $84.1 \%$ of countries among secondary+ educated women. The utilization of institutional delivery services was higher among wealthiest, urban, and secondary+ educated women compared to their counterparts. Greater utilization of private facilities for delivery was observed in women from the highest income group and urban communities, whereas highest utilization of public facilities was observed for women from the lowest income group and rural communities.

Conclusions: The utilization of institutional delivery services varied substantially between and within countries over time. Significant disparities in service utilization identified in this study highlight the need for tailored support for women from disadvantaged and vulnerable groups.

\section{INTRODUCTION}

nstitutional delivery is a necessary intervention to reduce delivery-related avoidable maternal and infant mortality. ${ }^{1}$ Between 1990 and 2015, more than 10 million women died globally due to pregnancy and childbirth-related complications. $^{2}$ Globally, 2.6 million newborns died in 2016, approximately 7,000 per day, ${ }^{3}$ and almost all $(99 \%)$ of these potentially preventable deaths occurred in low- and middle-income countries 
(LMICs). ${ }^{4}$ Pregnancy-related complications that lead to maternal mortality may occur during or shortly after childbirth. ${ }^{5}$ In LMICs, direct obstetric complications during childbirth were responsible for $70 \%$ of maternal deaths. ${ }^{6}$ Timely access to facility-based births save the lives of many mothers and newborns. ${ }^{7}$ In high-income countries, maternal mortality can be further reduced with increased rates of institutional delivery. ${ }^{8}$ In many LMICs, due to the geographical barriers in accessing services and the presence of cultural issues, women are accustomed to delivering babies at home, which leads to low utilization of institutional delivery services. To ensure equitable and accessible institutional delivery services, identifying vulnerable groups and populations within countries is crucial so that customized interventions can be developed and delivered.

The United Nations' Millennium Development Goals (MDGs) had a priority to improve maternal health and had set a target of reducing maternal mortality by three-quarters between 1990 and 2015 (MDG 5, target 5.A). ${ }^{9}$ Several initiatives have been introduced to achieve this target, including increased utilization of institutional delivery services. ${ }^{4}$ Earlier evidence showed improvements in the coverage of institutional delivery services in LMICs during the MDG era. ${ }^{10}$ During the same period, the world made remarkable progress in reducing maternal mortality by $43.9 \%$ from 385 deaths per 100,000 live births in 1990 to 216 in $2015 .^{2}$ However, this progress was uneven across countries and different populations within countries, and significant progress gaps consequently exist between populations.

To reduce such gaps, the global agenda shifted from MDGs to Sustainable Development Goals (SDGs). The highest priority of the SDG targets (target 3.8) is achieving universal health coverage (UHC), which means "all individuals and communities receive the health services they need without suffering financial hardship."11 Given the role of financial hardship in service utilization, it is also important to know which facility services (public or private) are increasing in LMICs and whether all people, irrespective of sociodemographic conditions, have equal access to these facilities. At the global level, evidence suggests an increasing trend in the utilization of institutional delivery services in sub-Saharan Africa, ${ }^{12,13}$ notably higher utilization by women from high-income groups residing in urban areas, ${ }^{14}$ as well as increasing use of private facilities for institutional delivery. ${ }^{15}$

However, comprehensive information is lacking on how socioeconomic and demographic disparities are associated with access to institutional delivery services, which limits the design of effective interventions/strategies required for equitable services. In addition, the extent to which these disparities are prevalent in public and private facilities remains unclear. Trend analysis at national and subpopulation levels helps policy makers and program managers assess overall progress, quantify gaps, and identify priority groups to guide strategies/interventions, further accelerating progress toward saving millions of lives of mothers and newborns. Therefore, this study aimed to examine the levels and trends in the utilization of institutional delivery services between LMICs and across subpopulations within LMICs.

\section{METHODS}

\section{Data}

This study used secondary data from large-scale, population-based, nationally representative repeated cross-sectional surveys conducted between 1990 and 2018 under the Demographic and Health Surveys (DHS) program. ${ }^{16}$ We extracted data from 74 LMICs across 5 DHS regions: sub-Saharan Africa (37 countries), South and Southeast Asia (12 countries), Central Asia (4 countries), North Africa-West AsiaEurope (10 countries), and Latin America and Caribbean (LAC; 11 countries). A detailed description of the surveyed country, survey year, and sample size is presented in the Supplement (Table S1).

\section{Outcome Variable}

The outcome variable in our study was institutional delivery. We used DHS standard recode files (KR files) to construct the variable for institutional delivery based on the responses of participants. The DHS provided information for institutional delivery for children born in the past 5 years in most of the countries. However, for some countries such as Bangladesh, the information on institutional delivery services was only available for children born in the past 3 years. Therefore, to allow crosscountry comparison, we defined institutional delivery services as the proportion of live births delivered in health facilities in the 3 years preceding the survey. We compared deliveries conducted in different types of health facilities (public versus private), and we particularly evaluated the proportion of deliveries that occurred in a public facility and those in a private facility. All calculations were conducted for live births.

\section{Statistical Analyses}

We estimated the weighted prevalence of institutional delivery services as proportions from the original survey data for all survey years of each

\section{A lack of information on how socioeconomic and demographic disparities affect access to institutional delivery services limits the design of effective interventions and strategies.}


study country. The rates of delivery in public and private facilities were estimated using the same method. However, we examined the geographical variation in the utilization of institutional delivery services during the latest DHS round. We calculated the variation in the utilization of institutional delivery services across subgroups in terms of place of residence, education of women, age of women, and wealth quintiles that the DHS constructed based on household assets by principal component analysis. ${ }^{17}$

For this study, we dichotomized education as below secondary (no or primary education) and secondary+ (secondary or higher) education. Similarly, we categorized age as 15-19 years (adolescents) and 20-49 years (adults). Also, we used place of residence (categorized as rural and urban) and wealth quintiles (categorized as poorest [first quintile], poorer, middle, richer, and richest [fifth quintile]) that the DHS provided with the survey data. Notably, we restricted our analysis to the country level but not at a regional level for 2 reasons. First, some regions (e.g., Central Asia) had data for a limited number of countries and heterogeneity between survey years (arbitrary). Second, we were interested in assessing progress across individual countries so that country-level programs and policies could be implemented.

To examine trends, a Bayesian linear regression model that used a Markov Chain Monte Carlo algorithm of multiple imputations for missing data was applied to estimate the institutional delivery rates and trends from 1990 to 2018 (Supplement). We reported $95 \%$ credible intervals (CrI) drawn from Bayesian analysis along with these estimates. We used the same technique to examine trends in the utilization of institutional delivery services across various sociodemographic groups to explore the changes in the utilization of institutional delivery services across sociodemographic subpopulations. We also validated our estimates drawn from regression models with those drawn from the original microdata (Supplement, Table S2).

To measure inequalities in the utilization of institutional delivery services, we applied both absolute and relative measure of inequalities. We estimated absolute inequality by subtracting the rate of the institutional delivery services in the poorest quintile from the rate of the institutional delivery services in the richest quintile, of rural from urban, of below secondary education from secondary+ education, and of adolescent mothers 15-19 years of age from adult mothers 20-49 years of age. We calculated rate ratio by dividing the rate of the institutional delivery services in the richest quintile by the rate of the institutional delivery services in the poorest quintile, and similarly the rate in urban by rural, in secondary+ education by below secondary education, and in adult mothers by adolescent mothers. To quantify the changes in inequalities over time, we measured changes in absolute and relative inequalities in the utilization of institutional delivery services from the earliest and latest rounds of DHS for countries that had at least 2 survey data points.

We used Stata (version 15.1) and R (version 3.5) statistical software to analyze the data.

\section{RESULTS}

\section{Sample Characteristics}

We included a total of 1,538,486 live births, from 256 surveys conducted in 74 countries, to assess whether these births took place at a health facility or at home. For trend analysis, we considered a total of 245 surveys conducted in 63 countries that had data on institutional delivery for at least 2 DHS rounds (Supplement, Table S1). Overall, 23.1\% of all live births were reported for women from the lowest quintile of wealth (poorest). The majority of birth data came from women living in rural areas $(67.5 \%)$ and women with below secondary education $(65.2 \%)$.

\section{Coverage in the Utilization of Institutional Delivery Services}

Our results show that the coverage of institutional delivery services varied between study countries (Figure 1). During 1990-2018, 19 of 74 countries reported that more than $90 \%$ of all deliveries were conducted at health facilities, with Armenia and Ukraine having universal coverage of institutional delivery services. In contrast, 13 countries had $<50 \%$ coverage of institutional delivery services, with the lowest in Chad $(23.7 \%)$ followed by Yemen (31.4\%) and Niger (33.1\%). Among all live births, the place of delivery (i.e., public or private health facilities) also varied across countries. In 52 of 74 countries, more than $50 \%$ of all live births took place in public health facilities. In comparison, 71 of 72 countries reported less than $50 \%$ of deliveries in private health facilities. The rate of public facilitybased delivery was highest in the Kyrgyz Republic (99.2\%) and lowest in Bangladesh (12.8\%). On the other hand, Egypt had the highest rate of private facility-based deliveries $(63.3 \%)$, whereas Tajikistan had the lowest $(0.1 \%)$. The rate of delivery in public health facilities was greater than delivery in private 
FIGURE 1. Geographical Variations in the Utilization of Institutional Delivery Services in Low- and MiddleIncome Countries During Latest Demographic and Health Survey Rounds ${ }^{a}$

\begin{tabular}{|c|c|}
\hline Delivery in public facility & Delivery in private facility \\
\hline Ukraine 2007 (100\%) & $3.0 \%$ \\
\hline Armenia $2016(100 \%)$ & $4.2 \%$ \\
\hline Maldives 2017 (99.7\%) & $21.2 \%$ \\
\hline Kyrgyz Republic 2012 (99.6\%) & $99.2 \%$ \\
\hline Albania 2018 (99.6\%) & $4.3 \%$ \\
\hline Jordan 2018 (99.2\%) & $34.1 \%$ \\
\hline Dominican Republic 2013 (99\%) & $28.3 \%$ \\
\hline Moldova 2005 (98.9\%) & $0.7 \%$ \\
\hline Kazakhstan 1999 (98.4\%) & $98.4 \%$ \\
\hline Turkey 2013 (98.3\%) & $38.6 \%$ \\
\hline Colombia $2015(97.2 \%)$ & $44.7 \%$ \\
\hline South Africa 2016 (96.3\%) & $7.8 \%$ \\
\hline Uzbekistan 1996 (94.1\%) & $0.0 \%$ \\
\hline Malawi 2016 (93.7\%) & $12.8 \%$ \\
\hline Brazil 1996 (93.5\%) & $79.8 \%$ \\
\hline Rwanda 2015 (93.1\%) & $0.8 \%$ \\
\hline Gabon 2012 (92.5\%) & $24.9 \%$ \\
\hline Republic of the Congo 2012 (92.1\%) & $14.7 \%$ \\
\hline Guyana 2009 (90.9\%) & $80.4 \%$ \\
\hline Burundi 2017 (89.3\%) & $3.1 \%$ \\
\hline Tajikistan 2017 (88.9\%) & $\mathbf{8 8 . 8 \%}$ \\
\hline Namibia 2013 (88.5\%) & $4.9 \%$ \\
\hline Egypt 2014 (88.2\%) & $24.6 \%$ \\
\hline Cambodia 2014 (87.3\%) & $15.9 \%$ \\
\hline Peru 2012 (87.2\%) & $9.5 \%$ 미 $77.3 \%$ \\
\hline Benin $2018(86.1 \%)$ & $14.6 \%$ \\
\hline Honduras $2012(84.9 \%)$ & $80.1 \%$ \\
\hline Philippines 2017 (83.7\%) & $25.7 \%$ \\
\hline Zimbabwe 2015 (81.6\%) & $69.3 \%$ \\
\hline India $2016(81.4 \%)$ & $26.9 \%$ \\
\hline Democratic Republic of the Congo 2014 (81.3\%) & $64.9 \%$ \\
\hline Indonesia 2017 (81.2\%) & $49.0 \%$ \\
\hline Sao Tome and Principe 2009 (81.1\%) & $80.9 \%$ \\
\hline Senegal $2017(80.8 \%)$ & $76.5 \%$ \\
\hline Azerbaijan 2006 (80.3\%) & $79.4 \%$ \\
\hline Vietnam 2002 (78.7\%) & $3.8 \%$ \\
\hline Lesotho 2014 (78.7\%) & $3.5 \%$ \\
\hline Comoros $2012(78.4 \%)$ & $76.1 \%$ \\
\hline Uganda 2016 (76.3\%) & $59.8 \%$ \\
\hline Swaziland $2007(75.5 \%)$ & $44.6 \%$ \\
\hline Ghana 2014 (75.5\%) & $67.2 \%$ \\
\hline Liberia 2016 (75.3\%) & $15.2 \%-60.1 \%$ \\
\hline Togo 2014 (75.1\%) & $12.2 \%-62.8 \%$ \\
\hline Zambia $2014(71.6 \%)$ & $5.2 \%-66.4 \%$ \\
\hline Mozambique 2015 (70.6\%) & $0.4 \% \quad 70.2 \%$ \\
\hline Pakistan 2018 (69.9\%) & $24.7 \%$ \\
\hline Bolivia 2008 (69.6\%) & $11.0 \%$ \\
\hline Nicaragua $2001(69.4 \%)$ & $62.5 \%$ \\
\hline Guatemala $2015(68 \%)$ & $59.4 \%$ \\
\hline Burkina Faso 2010 (66.7\%) & $65.7 \%$ \\
\hline Kenya 2014 (64.9\%) & $16.3 \%$ \\
\hline Tanzania $2016(64.7 \%)$ & $12.5 \%$ \\
\hline Gambia $2013(63.7 \%)$ & $58.2 \%$ \\
\hline Cameroon $2011(63.5 \%)$ & $22.6 \%$ \\
\hline Morocco 2004 (63.4\%) & $56.0 \%$ \\
\hline Nepal 2016 (62.1\%) & $11.5 \%$ \\
\hline Cote d'Ivoire 2012 (59.8\%) & $4.9 \%$ \\
\hline Mali $2013(58.6 \%)$ & $55.8 \%$ \\
\hline Sierra Leone 2013 (57.8\%) & $55.8 \%$ \\
\hline Paraguay 1990 (54.8\%) & $14.2 \%$ \\
\hline Central African Republic 1995 (50.2\%) & $47.0 \%$ \\
\hline Timor-Leste 2016 (50\%) & $48.7 \%$ \\
\hline Afghanistan 2015 (48.6\%) & $43.4 \%$ \\
\hline Angola $2016(47.4 \%)$ & \begin{tabular}{l|l}
$1.7 \%$ & $45.6 \%$
\end{tabular} \\
\hline Myanmar $2016(43 \%)$ & $6.9 \%=36.1 \%$ \\
\hline Guinea $2012(41.2 \%)$ & $5.0 \%-36.2 \%$ \\
\hline Haiti 2017 (39.2\%) & $4.7 \% \quad 34.6 \%$ \\
\hline Bangladesh 2014 (37.7\%) & $22.4 \%-12.8 \%$ \\
\hline Nigeria 2013 (37.4\%) & $13.7 \%-23.7 \%$ \\
\hline Madagascar 2009 (35.1\%) & $2.7 \%$ \\
\hline Ethiopia 2016 (33.3\%) & $1.1 \% \quad 31.9 \%$ \\
\hline Niger 2012 (33.1\%) & $0.5 \% \quad 32.6 \%$ \\
\hline Yemen 2013 (31.4\%) & $11.4 \%-20.0 \%$ \\
\hline Chad $2015(23.7 \%)$ & $1.1 \% 22.6 \%$ \\
\hline
\end{tabular}




\section{During 1990- 2018, the utilization of institutional delivery services increased in 60 of 63 countries, but the progress varied across countries.}

health facilities in all countries, except Bangladesh, Egypt, Indonesia, and Pakistan.

\section{Trends in the Utilization of Institutional Delivery Services}

During 1990-2018, the utilization of institutional delivery services increased in 60 of 63 study countries (Figure 2). The progress in the utilization of institutional delivery services varied across countries. The highest increase in the utilization of institutional delivery services was observed in Cambodia (an $18.3 \%$ annual increase from $0.6 \%$ in 1990 to $94.0 \%$ in 2018 ) followed by Sierra Leone (16.2\%) and Timor-Leste (13.7\%). At the same time, utilization decreased in Angola $(-0.9 \%)$, Kazakhstan $(-0.3 \%)$, and Madagascar $(-1.4 \%)$. The increase in the utilization of institutional delivery services steadily decreased after 1990-1999 in most LMICs (Figure 2). Based on this trend, 31 of 63 countries were estimated to have $<80 \%$ utilization of institutional delivery services in 2018 , with the highest in Armenia (100\%, 95\% CrI 100\%-100\%) and lowest in Chad $(26.1 \%$, 95\% CrI 15.3\%-38.7\%) (Figure 3).

Trends in the utilization of institutional delivery services varied across wealth, residence, education, and age of mother over time. From 1990 to 2018, the utilization of institutional delivery services in the lowest income group increased in $90.3 \%$ of countries ( 56 of 62 countries), with the highest in Cambodia (27.7\% increase). In comparison, utilization declined in $9.7 \%$ of countries ( 6 of 62 countries), with the largest decline being $-6.1 \%$ in Nigeria. Over $90.3 \%$ of countries (56 of 62 countries) reported increasing utilization of institutional delivery services by the highest income group, with the highest increase seen in Sierra Leone (14.4\%), and $9.7 \%$ of countries ( 6 of 62 countries) showed a decline in the utilization of institutional delivery services with the largest decline in Angola $(-0.6 \%)$ (Supplement, Table S3). If this trend continues, Nigeria $(4.7 \%$, 95\% CrI 1.9\%-9.6\%) and Yemen $(47.0 \%, 95 \%$ CrI $0.0 \%-95.8 \%)$ are estimated to have the lowest utilization of institutional delivery services in the lowest and highest income groups, respectively (Supplement, Table S4).

During the same time in rural areas, the utilization of institutional delivery services increased in $93.7 \%$ of countries (59 of 63 countries), with the highest increase by $20.5 \%$ in Cambodia, and $6.3 \%$ of countries (4 of 63 countries) reported a decline in utilization, with the largest decline

FIGURE 2. Change Rates of Institutional Delivery Services in Low- and Middle-Income Countries
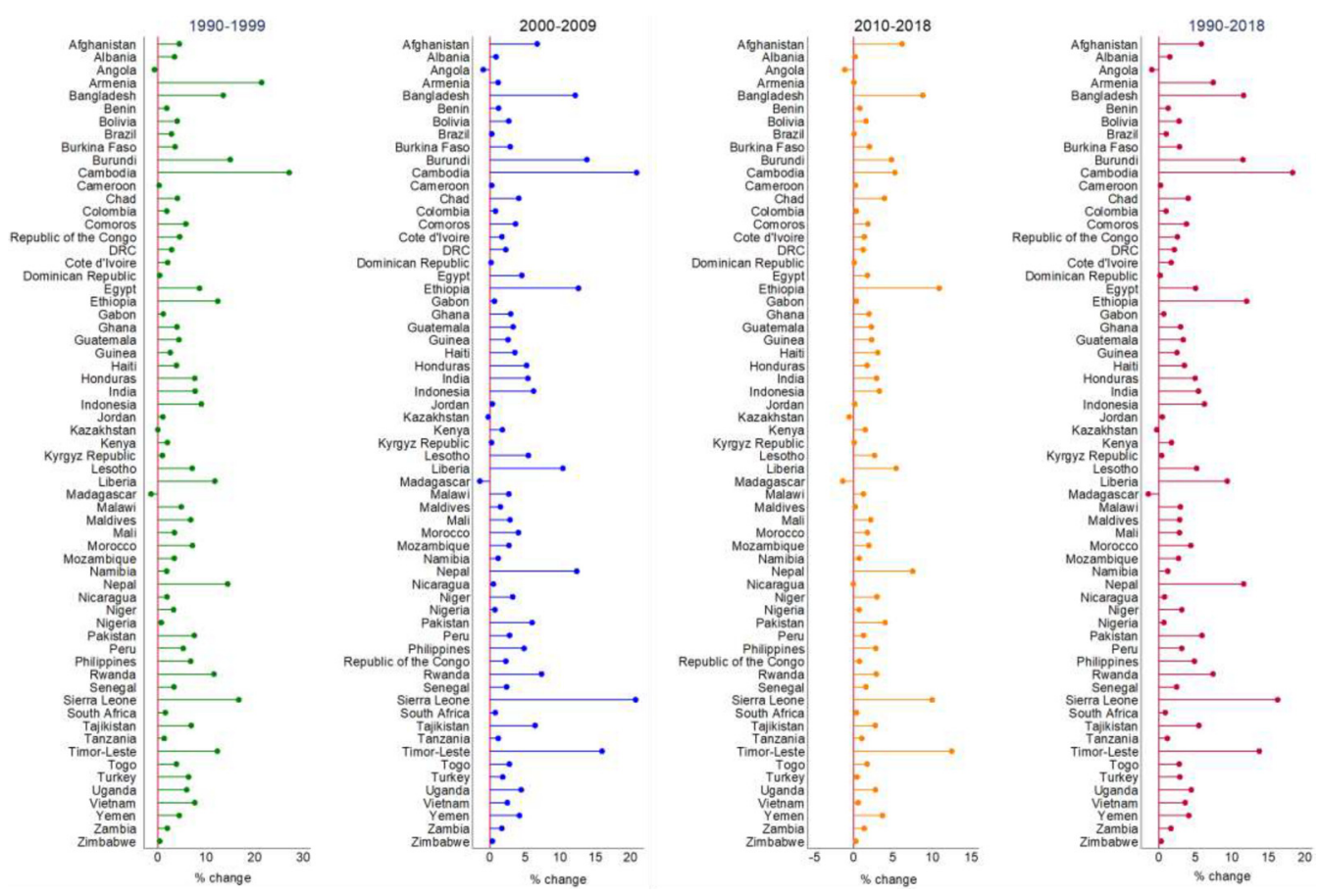
FIGURE 3. Trends in the Utilization of Institutional Delivery Services in Low- and Middle-Income Countries

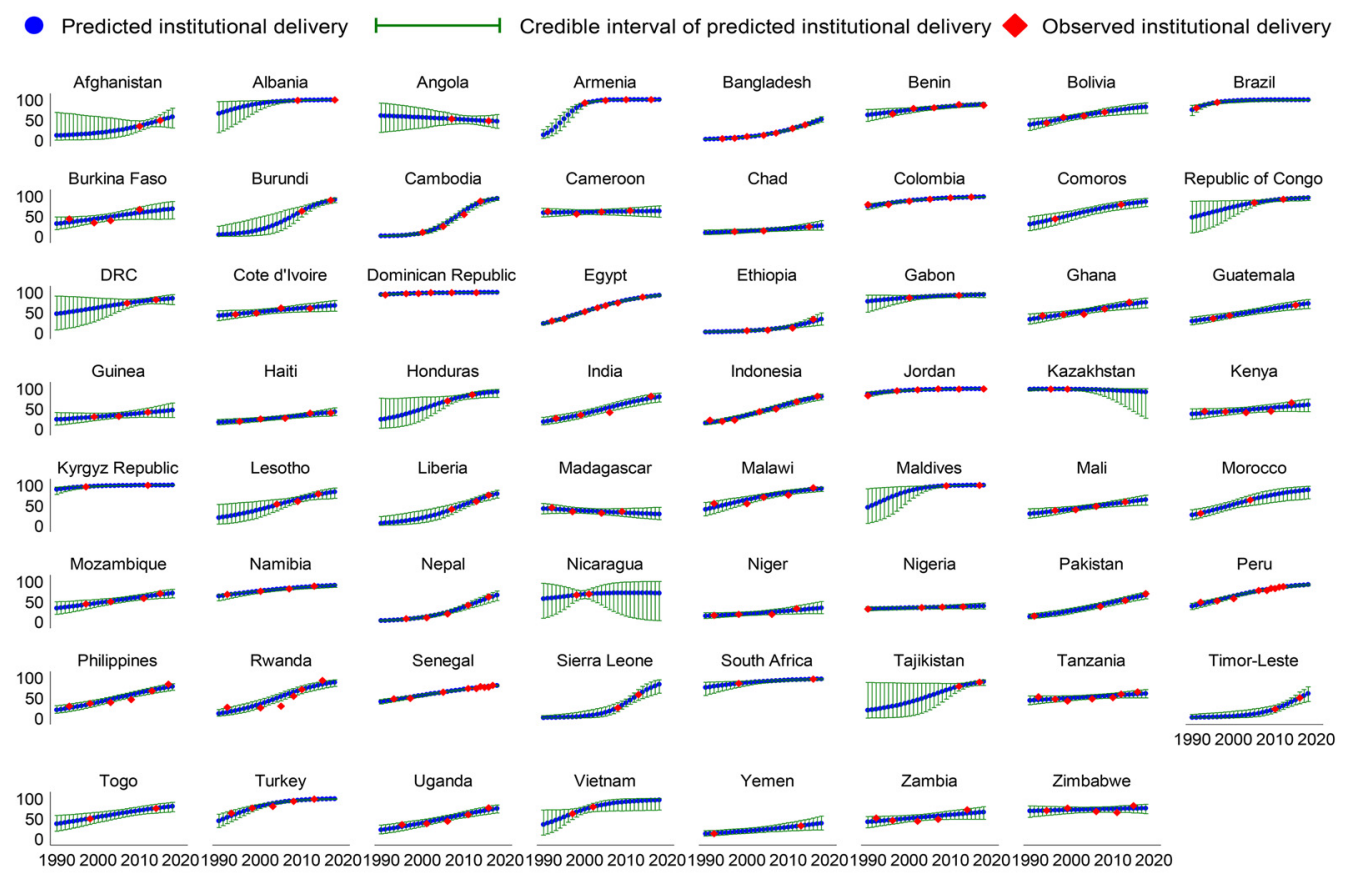

observed in Angola (-2.1\%). In contrast, in urban areas, $95.2 \%$ of countries (60 of 63 countries) experienced an increasing rate of institutional delivery services, with the highest increase in Cambodia ( $14.5 \%$ ), and $4.8 \%$ of countries ( 3 of 63 countries) showed a decline in the utilization of institutional delivery services, with the largest decline in Angola by $-1.4 \%$ (Supplement, Table S5). Similar to trends related to wealth and place of residence, the utilization of institutional delivery services also varied over time across women's education (Supplement, Tables S7 and S8) and age (Supplement, Tables S9 and S10).

\section{Changes in Inequalities in the Utilization of Institutional Delivery Services}

Among 60 countries, inequalities in the utilization of institutional delivery services increased in relation to wealth, place of residence, age, and education. Wealth-related inequalities widened in 16 countries during the latest DHS round compared with the earliest, with the highest increase of $41.4 \%$ occurring in Ethiopia (earliest round: poorest $0.8 \%$, richest $22.9 \%$; latest round: poorest $13.9 \%$, richest $77.5 \%$ ) (Figure 4 ). In terms of place of residence, 10 of 63 countries experienced a growing gap in this inequality, with the highest increase of $29.4 \%$ occurring in Ethiopia (earliest round: rural $1.8 \%$, urban $32.5 \%$; latest round: rural $26.3 \%$, urban $86.3 \%$ ) (Supplement, Figure S1). Among these countries, a widening in the inequality of institutional delivery service utilization was seen in 10 countries in terms of education, with the highest increase of $20.2 \%$ in Madagascar (Supplement, Figure S2), and in 36 countries in terms of age, with the highest increase of $13.9 \%$ in Burundi (Supplement, Figure S3). In some countries, utilization of institutional delivery services increased among the advantaged groups and decreased among the disadvantaged groups during the latest round of surveys (Supplement, Figures S1-S3). Relative inequalities in the utilization of institutional delivery services also changed during the earliest and latest DHS rounds across wealth, place of residence, education, and age of women (Supplement, Tables S11-S14).

\section{Changes in Institutional Delivery Between Public and Private Facilities}

We explored the variations in the utilization of institutional delivery services by the type of facilities (i.e., public and private health facilities) to understand

\author{
In many study \\ countries, \\ inequalities in the \\ utilization of \\ institutional \\ delivery services \\ increased in \\ relation to wealth, \\ place of residence, \\ age, and \\ education.
}


FIGURE 4. Changes of Inequalities in the Utilization of Institutional Delivery Services Between Earliest and Latest Time Points in Low- and Middle-Income Countries by Wealth Quintiles
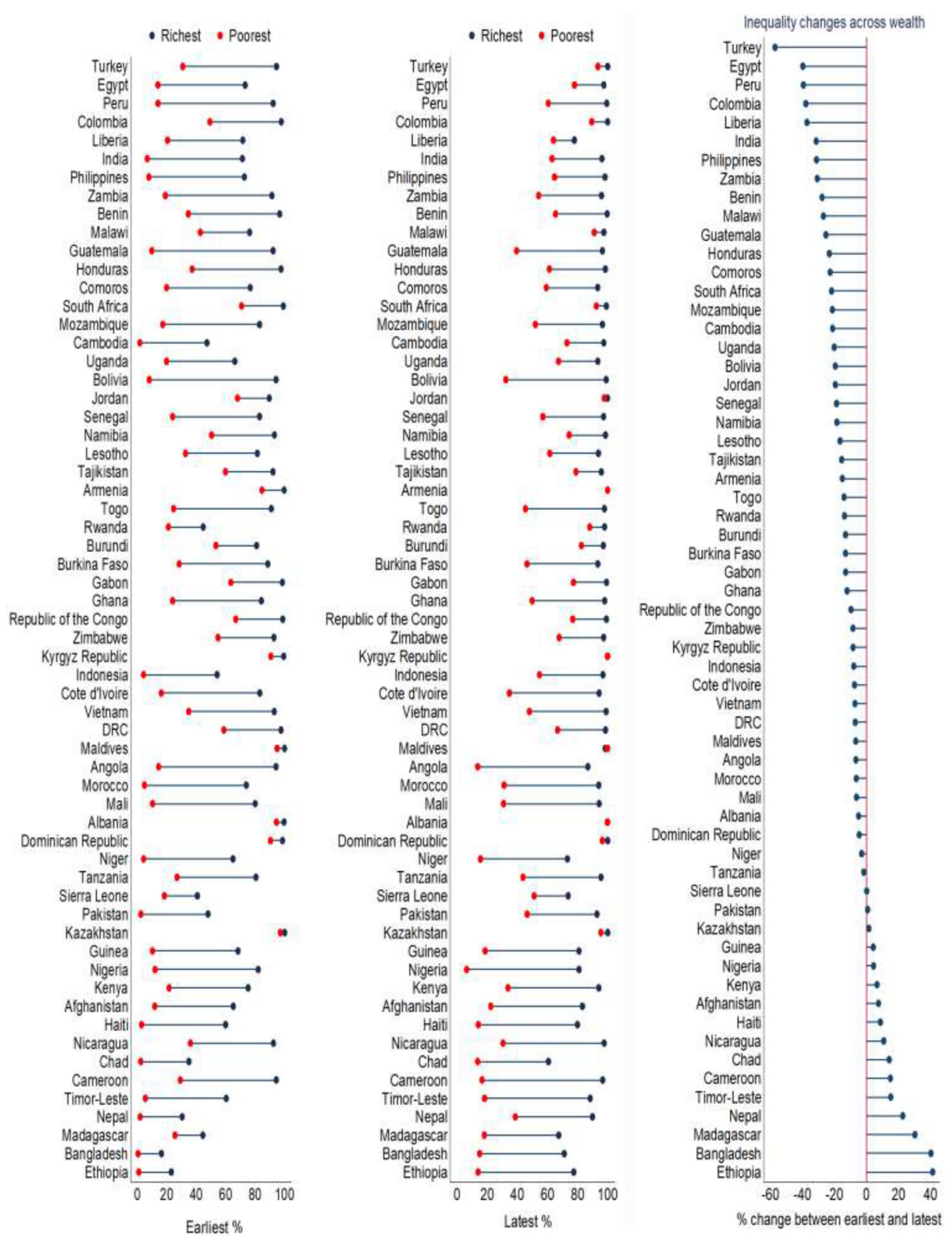

the differences in service provision (Supplement, Table S15 and Figure 5). Although an increase in the utilization of institutional delivery services was observed, this increase was common across public facilities in 54 countries and private facilities in 43 countries. During 1990-2018, the highest increase in the utilization of institutional delivery services was observed in Sierra Leone (16.8\%) in public facilities 
FIGURE 5. Trends in the Utilization of Delivery Services Facilitated by Public and Private Sectors in Low- and Middle-Income Countries

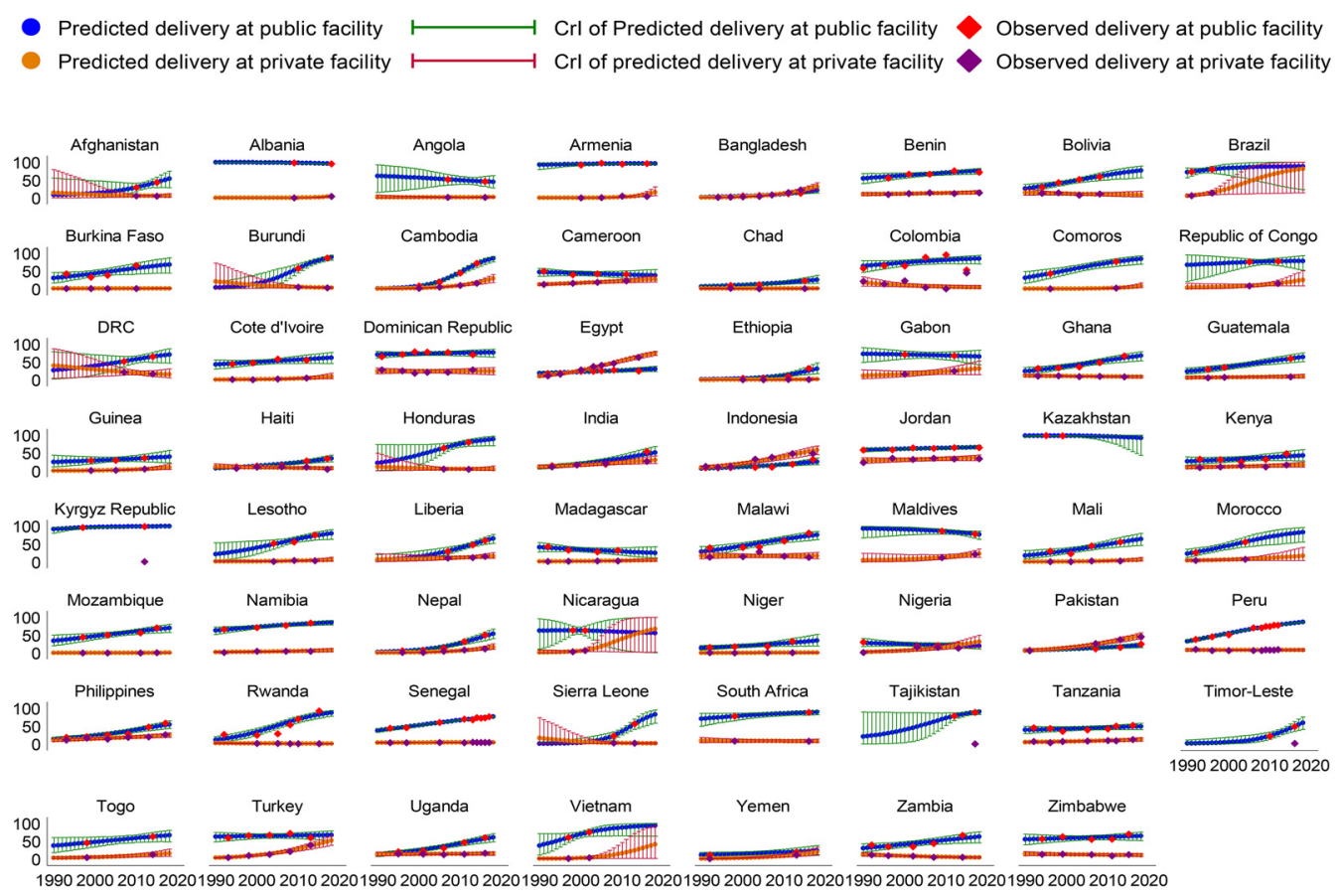

and Albania (30.2\%) in private facilities. During the same period, the utilization of institutional delivery services decreased in some countries in both public and private facilities, with the largest declines in Madagascar $(-1.7 \%)$ in public facilities and Sierra Leone $(-8.1 \%)$ in private facilities.

Significant disparities exist in the utilization of delivery services in public and private facilities between countries and across wealth quintiles, residence, education, and age of women within countries. In most of the countries, the delivery in both public and private facilities was mostly dominated by the richest rather than the poorest women (Supplement, Tables S16 and S17). However, these gaps across residence, education, and age are minimal in most countries. (Supplement, Tables S18-S23).

Change rates in the utilization of delivery services in public and private facilities varied between countries, between periods within countries, and between countries and periods across wealth quintiles, residence, education, and age. In public facilities, the increase in the utilization of delivery services was highest in Cambodia $(27.9 \%)$ among the poorest, and in Sierra Leone (17.2\%) among the richest (Supplement, Table S24). Whereas,
Cambodia (24.0\%) and Albania (33.6\%) had the highest increase in the utilization of delivery services in private facilities among the poorest and the richest groups, respectively (Supplement, Table S25). Variations in the utilization of delivery services were also apparent across the place of residence, education, and age in both public and private facilities (Supplement, Tables S26-S31).

\section{DISCUSSION}

During the latest DHS round, the utilization of institutional delivery services varied substantially across countries and over time. The utilization across public and private health facilities was not uniform across countries. Among study LMICs, Significant 16 countries had $\geq 80 \%$ utilization of delivery ser- disparities exist in vices in public facilities, whereas no countries had the utilization of $\geq 80 \%$ utilization of this service in private facilities delivery services during the latest DHS rounds. Trend analysis in public and showed a sustained increase in the utilization of private facilities institutional delivery services in most countries. between countries Our findings showed a significant influence of and across specific wealth quintile, place of residence, and education populations within of women in the utilization of institutional countries. 


\section{Our results highlight uneven progress in the utilization of institutional delivery services between countries and across subpopulations within countries.}

delivery services. In many countries, the service utilization gaps observed in the earliest DHS rounds were found to be persistent and widened further in the latest DHS rounds.

Geographical variations in the utilization of institutional delivery services were expectedly common and were in agreement with previous studies. ${ }^{18}$ During the latest DHS rounds, in nearly $20 \%$ of countries included in our analysis, less than half of deliveries took place at a health facility. This finding highlights that still more than half of the babies are delivered at home in many countries such as Chad, Yemen, and Niger. Traditional and familial influences, distance to the facility, cost of delivery, perceptions of low quality of care, and fear of discrimination play a key role in inadequate utilization of facility-based delivery. ${ }^{19}$

Our findings on increasing trends in the utilization of institutional delivery services are consistent with previous studies. ${ }^{12}$ However, our results also highlight uneven progress in the utilization of institutional delivery services between countries and across subpopulations within countries. The utilization of institutional delivery services decreased by nearly 1.5 percentage points in Madagascar.

The presence of disparities in the utilization of institutional delivery services across income and education levels is supported by previous research. ${ }^{14,20}$ We found lower utilization of institutional delivery services among women of the poorest quintile (lowest income group). Similar to previous research, we also found that compared with their counterparts, women from the lowest income group have lower access to private facilities for delivery. ${ }^{13,15,21}$ We also identified countries such as Bangladesh where inequality in the utilization of institutional delivery services is further increased. In particular, wealth-based inequalities in the utilization of institutional delivery services widened in 19 countries, while residence- and education-based inequalities grew further in 10 countries each. This finding highlights the need of revisiting strategies and implementing appropriate interventions to reduce the inequalities in the utilization of institutional delivery services across various sociodemographic groups.

Our study demonstrates an increasing trend in the majority of countries toward greater utilization of public health facilities for delivery. The predominant role of public facilities in increased utilization of institutional delivery services was proven in previous studies. ${ }^{13}$ The reasons for using public facilities for delivering births could be multifaceted. The lower delivery cost is reported to greatly influence the use of this service. ${ }^{22,23}$ Also, increasing the number of health care providers through recruitment and improving the quality of care by training frontline health service providers are the key factors driving the growing rates of delivery in public facilities. ${ }^{24-27}$ However, compared with public facility-based deliveries, the rate of deliveries in private facilities was greater in Egypt, Indonesia, Pakistan, and Bangladesh by $38.6,29.4,20.5$, and 9.6 percentage points respectively. The higher rate of deliveries in private facilities could be due to better quality of services, shorter wait time, higher availability of health care providers, greater privacy, and the visualization of social status. ${ }^{28}$ Affordability and availability of private services are also increased due to the growth of gross domestic product per capita in these $^{\text {countries. }}{ }^{29}$ In general, an increase in awareness about the benefit of facility-based delivery might be the key to the increased utilization of institutional delivery services. ${ }^{12}$ This may further result in the reduction of maternal and newborn mortality. However, we acknowledge that merely moving births to health facilities does not eliminate maternal and child mortality.

Our analysis has shown that most LMICs have reported remarkable improvement in the utilization of institutional delivery services. For example, the LAC countries showed the highest utilization of institutional delivery services. Innovative strategies have helped reduce financial barriers to access maternal health care in LAC countries; these include national health insurance (Brazil, Chile, Colombia, Jamaica, Mexico, and Peru), free health insurance scheme for lower-income families (Bolivia, Mexico, and Peru), incorporating UHC as a constitutional right (Brazil and Chile), and public-private partnerships (Colombia). ${ }^{30}$ Reducing the gap in provisioning institutional delivery services for a particular demographic group, such as the one for indigenous and African origin women, was also considered there. ${ }^{30}$ Some vertical approaches can also be attributed to the growing rates of institutional delivery services utilization in Asian countries. For example, conditional cash incentives in India ${ }^{31}$ and demandside financing in Bangladesh ${ }^{32}$ are linked with higher utilization of institutional delivery services.

Higher rates of home delivery assisted by a traditional birth attendant are common in many settings. Restricting the services from a traditional birth attendant backed up by hospital readiness increased facility-based births from $<30 \%$ during the start of MDG era to the current rate of $>90 \%$ rate in 
Malawi and Rwanda. ${ }^{33-36}$ In contrast, countries with slower progress or decreasing trends of utilizing institutional delivery services such as Angola, Kazakhstan, and Madagascar have shown higher inequality in the utilization of institutional delivery services. Distance to health facilities, lower educational level, and rural residence were the major determinants of poor utilization of this service in sub-Saharan African countries. ${ }^{7}$ Moreover, the current coronavirus disease (COVID-19) pandemic situation can aggravate the poor utilization of institutional delivery services because people may not be accessing health facilities as they would have before COVID.

The major strength of this study is the use of population-based nationally representative samples covering both rural and urban areas of 74 LMICs and the identification of population subgroups within LMICs. Analysis at the subpopulation level is particularly helpful to design interventions for target populations.

\section{Limitations}

The use of the same standard methodology across countries allows cross-country comparison of the estimates. However, older and fewer data points created wider credible intervals of the estimates in some countries (e.g., Kazakhstan). Credible intervals could be smaller for countries with many data points (e.g., Bangladesh). Estimates drawn from authentic representative data collected from multiple sources may better predict the indicators with lower uncertainty. Moreover, the DHS data are mostly self-reported and hence are prone to recall bias. However, the DHS has followed a standard methodology and questionnaire for more than 3 decades to provide population-based data that are comparable and representative not only at the national level but also subnational and subpopulation levels.

\section{CONCLUSIONS}

Although the utilization of institutional delivery services varied substantially across LMICs, the utilization of health facilities for delivery overall increased in most of the countries between 1990 and 2018. However, this increase was not uniform across countries and sociodemographic subpopulations (e.g., poorest and richest, and rural and urban) within countries. Unfortunately, inequalities in the utilization of institutional delivery services are widening in some countries. These findings warrant the development of appropriate and tailored interventions covering the disadvantaged and marginalized populations identified in this study to achieve the global target of "leaving no one behind" for the utilization of institutional delivery services by 2030 .

Acknowledgments: We thank the Demographic and Health Survey program for providing access to the data sets. We gratefully acknowledge the commitment of the Australian Government and the University of Queensland, Brisbane, QLD, Australia, to their research efforts. To undertake the PhD degree, MMH is supported by the "Research Training Program" scholarship funded by the Commonwealth Government of Australia and the University of Queensland, Brisbane, QLD, Australia.

Author contributions: $\mathrm{MMH}$ conceptualized the study plan, designed the study, executed the data, managed the data, compiled and prepared final data set for analysis, analyzed the data, interpreted the results, and wrote the manuscript. RJSM, SA, and AAM provided guidance in conducting the study. YF contributed to interpreting the findings and drafting and revising the manuscript. RJSM, YF, SA, and AAM critically reviewed the analysis and final version of the manuscript. All authors read and approved the final manuscript for publication.

Competing interests: None declared

\section{REFERENCES}

1. Moyer CA, Adanu RM, Engmann CM. The relationship between facility-based delivery and maternal and neonatal mortality in SubSaharan Africa. Int J Gynaecol Obstet. 2013;122(3):263-265. CrossRef. Medline

2. Alkema L, Chou D, Hogan D, et al. Global, regional, and national levels and trends in maternal mortality between 1990 and 2015, with scenario-based projections to 2030: a systematic analysis by the UN Maternal Mortality Estimation Inter-Agency Group. Lancet. 2016;387(10017):462-474. CrossRef. Medline

3. United Nations Children's Fund (UNICEF), World Health Organization, World Bank, United Nations. Levels \& Trends in Child Mortality: Report 2017. UNICEF; 2017. Accessed January 7, 2021. https://childmortality.org/wp-content/uploads/2018/10/UNIGME-Child-Mortality-Report-2017.pdf

4. World Health Organization (WHO). Trends in Maternal Mortality: 1990 to 2015: Estimates by WHO, UNICEF, UNFPA, World Bank Group and the United Nations Population Division. WHO; 2015. Accessed January 7, 2021. https://www.who.int/reproductive health/publications/monitoring/maternal-mortality-2015/en/

5. Ronsmans C, Graham WJ; Lancet Maternal Survival Series steering group. Maternal mortality: who, when, where, and why. Lancet. 2006;368(9542):1189-1200. CrossRef. Medline

6. Ronsmans C, Campbell OMR, McDermott J, Koblinsky M. Questioning the indicators of need for obstetric care. Bull World Health Organ. 2002;80(4):317-324. Medline

7. Rosário EVN, Gomes MC, Brito M, Costa D. Determinants of maternal health care and birth outcome in the Dande Health and Demographic Surveillance System area, Angola. PLoS One. 2019; 14(8):e0221280. CrossRef. Medline

8. Koblinsky MA, Campbell O, Heichelheim J. Organizing delivery care: what works for safe motherhood? Bull World Health Organ. 1999;77(5):399-406. Medline

9. United Nations. The Millennium Development Goals Report 2015. United Nations; 2015. Accessed January 7, 2021. https://www.un. org/en/development/desa/publications/mdg-report-2015.html

10. ICF. DHS final reports. Accessed January 7, 2021. https://dhs program.com/publications/publication-search.cfm?type $=5$

11. World Health Organization (WHO). Universal health coverage (UHC). Accessed January 7, 2021. https://www.who.int/newsroom/fact-sheets/detail/universal-health-coverage-(uhc) 
12. Doctor HV, Nkhana-Salimu S, Abdulsalam-Anibilowo M. Health facility delivery in sub-Saharan Africa: successes, challenges, and implications for the 2030 development agenda. BMC Public Health 2018;18(1):765. CrossRef. Medline

13. Doctor HV, Radovich E, Benova L. Time trends in facility-based and private-sector childbirth care: analysis of Demographic and Health Surveys from 25 sub-Saharan African countries from 2000 to 2016. J Glob Health. 2019;9(2):020406. CrossRef. Medline

14. Joseph G, da Silva ICM, Wehrmeister FC, Barros AJD, Victora CG. Inequalities in the coverage of place of delivery and skilled birth attendance: analyses of cross-sectional surveys in 80 low and middleincome countries. Reprod Health. 2016;13(1):77. CrossRef. Medline

15. Benova L, Macleod D, Footman K, Cavallaro F, Lynch CA, Campbell OMR. Role of the private sector in childbirth care: cross-sectional survey evidence from 57 low- and middle-income countries using Demographic and Health Surveys. Trop Med Int Health. 2015;20 (12): 1657-1673. CrossRef. Medline

16. Rutstein SO, Rojas G. Guide to DHS Statistics. Demographic and Health Surveys, ORC Macro; 2006. Accessed January 7, 2021 https://dhsprogram.com/pubs/pdf/DHSG1/Guide_to_DHS Statistics_29Oct2012_DHSG1.pdf

17. Rutstein SO, Johnson K. DHS Comparative Reports No. 6 The DHS Wealth Index. ORC Macro; 2004. Accessed January 7, 2021. https://dhsprogram.com/publications/publication-cr6comparative-reports.cfm

18. Kanyangarara $M$, Chou VB, Creanga AA, Walker N. Linking household and health facility surveys to assess obstetric service availability, readiness and coverage: evidence from 17 low- and middle-income countries. J Glob Health. 2018;8(1):010603 CrossRef. Medline

19. Bohren MA, Hunter EC, Munthe-Kaas HM, Souza JP, Vogel JP Gülmezoglu AM. Facilitators and barriers to facility-based delivery in low- and middle-income countries: a qualitative evidence synthesis. Reprod Health. 2014;11(1):71. CrossRef. Medline

20. Lohela TJ, Nesbitt RC, Pekkanen J, Gabrysch S. Comparing socioeconomic inequalities between early neonatal mortality and facility delivery: cross-sectional data from 72 low- and middle-income countries. Sci Rep. 2019;9(1):9786. CrossRef. Medline

21. Victora CG, Joseph G, Silva ICM, et al. The inverse equity hypothesis: analyses of institutional deliveries in 286 national surveys. Am J Public Health. 2018;108(4):464-471. CrossRef. Medline

22. Meessen $B$, Hercot $D$, Noirhomme $M$, et al. Removing user fees in the health sector: a review of policy processes in six sub-Saharan African countries. Health Policy Plan. 2011;26(Suppl 2):ii16-ii29. CrossRef. Medline

23. Nabyonga Orem J, Mugisha F, Kirunga C, Macq J, Criel B. Abolition of user fees: the Uganda paradox. Health Policy Plan. 2011;26 (Suppl 2):ii41-ii51. CrossRef. Medline
24. Nyonator FK, Awoonor-Williams JK, Phillips JF, Jones TC, Miller RA The Ghana Community-based Health Planning and Services Initiative for scaling up service delivery innovation. Health Policy Plan. 2005;20(1):25-34. CrossRef. Medline

25. Doctor HV, Findley SE, Ager A, et al. Using community-based research to shape the design and delivery of maternal health services in Northern Nigeria. Reprod Health Matters. 2012;20(39):104-1 12. CrossRef. Medline

26. Ramsey K, Hingora A, Kante M, et al. The Tanzania Connect Project: a cluster-randomized trial of the child survival impact of adding paid community health workers to an existing facility-focused health system. BMC Health Serv Res. 2013;13(S2)(Suppl 2):S6. CrossRef. Medline

27. Sherr K, Cuembelo F, Michel C, et al. Strengthening integrated primary health care in Sofala, Mozambique. BMC Health Serv Res 2013;13(S2)(Suppl 2):S4. CrossRef. Medline

28. Wong KLM, Radovich E, Owolabi OO, et al. Why not? Understanding the spatial clustering of private facility-based delivery and financial reasons for homebirths in Nigeria. BMC Health Serv Res. 2018;18(1):397. CrossRef. Medline

29. The World Bank. DataBank: World Development Indicators. World Bank; 2020. Accessed January 7, 2021. https://databank. worldbank.org/source/world-development-indicators\#

30. Montagu D, Sudhinaraset M, Diamond-Smith N, et al. Where women go to deliver: understanding the changing landscape of childbirth in Africa and Asia. Health Policy Plan. 2017;32(8):1146-1 152. CrossRef. Medline

31. Randive B, Diwan V, De Costa A. India's conditional cash transfer programme (the JSY) to promote institutional birth: is there an association between institutional birth proportion and maternal mortality? PLoS One. 2013;8(6):e67452. CrossRef. Medline

32. Anwar I, Nababan HY, Mostari S, Rahman A, Khan JAM Trends and inequities in use of maternal health care services in Bangladesh, 1991-2011. PLoS One. 2015;10(3):e0120309. CrossRef. Medline

33. Cammack D. Local governance and public goods in Malawi. IDS Bull. 2011;42(2). CrossRef

34. Bucagu M, Kagubare JM, Basinga P, Ngabo F, Timmons BK, Lee AC. Impact of health systems strengthening on coverage of maternal health services in Rwanda, 2000-2010: a systematic review. Reprod Health Matters. 2012;20(39):50-61. CrossRef. Medline

35. Campbell OMR, Calvert $C$, Testa A, et al. The scale, scope, coverage, and capability of childbirth care. Lancet. 2016;388(10056):21932208. CrossRef. Medline

36. Chitsa Banda E. Stakeholders' Perceptions of the Changing Role of Traditional Birth Attendants in The Rural Areas of Central West Zone, Malawi: A Mixed Methods Study. PhD thesis. University of the Witwatersrand, Johannesburg; 2013.

\section{Peer Reviewed}

Received: September 4, 2020; Accepted: December 15, 2020; First published online: February 10, 2021

Cite this article as: Hasan MM, Magalhaes RJS, Fatima Y, Ahmed S, Mamun AA. Levels, trends, and inequalities in using institutional delivery services in low- and middle-income countries: a stratified analysis by facility type. Glob Health Sci Pract. 2021 ;9(1):78-88. https://doi.org/10.9745/GHSP-D20-00533

(C) Hasan et al. This is an open-access article distributed under the terms of the Creative Commons Attribution License, which permits unrestricted use distribution, and reproduction in any medium, provided the original author and source are properly cited. To view a copy of the license, visit https:// creativecommons.org/licenses/by/4.0/. When linking to this article, please use the following permanent link: https://doi.org/10.9745/GHSP-D-2000533 